\title{
Asymmetric Synthesis of Nodulones C \& D by Chemoenzymatic Ap- proach gives Insight into their Biosynthesis
}

\author{
Tanaya Manna, Anshul Rajput, Nirmal Saha, Amit Mondal, Syed Masood Husain* \\ Centre of Biomedical Research, Sanjay Gandhi Postgraduate Institute of Medical Sciences Campus, Raebareli Road, Lucknow \\ 226014, India
}

Supporting Information Placeholder

\begin{abstract}
The first asymmetric total synthesis of fungal secondary metabolites, $(R)$-nodulone $\mathrm{C}(\mathbf{4})$ and trans-nodulone $\mathrm{D}(\mathbf{5})$ has been reported through the chemoenzymatic approach. The strategy utilizes NADPH-dependent naphthol reductases of Magnaporthe grisea for the reduction of putative biosynthetic substrates, synthesized non-enzymatically in multiple steps. A dihydronaphthalenone 32 and cis-nodulone D (30) has also been synthesized chemoenzymatically. The work implies for similar steps during the biosynthesis of nodulones and their analogs with the involvement of tetrahydroxynaphthalene reductase related enzyme(s).
\end{abstract}

Endophytic fungi are known to produce a large number of secondary metabolites with diverse structural features, which display a wide array of biological activities. ${ }^{1-3}$ Among these, nodulones 1-6 belongs to a new class of bioactive polyketides produced by Nodulisporium sp., a group of common endophytic fungi harboured by many plants (Fig. 1). Isofuranonaphthalenone 1, a tricyclic compound has been isolated from Nodulisporium $s p$. A4 from Aquilaria sinensis and was mistakenly assigned cisconfiguration. ${ }^{4}$ The stereochemistry was later found to be $(7 S, 8 S)$ based on the X-ray crystallographic analysis of $\mathbf{1}$, isolated from Daldinia eschscholtzii and is named here as trans-nodulone A (1) ${ }^{5}$ It shows immunosuppressive activity and cytotoxicity against tumor cells SF-268..$^{4-5}$ In addition, the cis-nodulone A (2) has also been isolated from Nodulisporium sp. of Antidesma ghaesmbilla and was found to display antimycobacterial and antimalarial activities with IC50 values of 3.125 and $11.3 \mu \mathrm{g} / \mathrm{ml}$, respectively. ${ }^{6}$ Related dihydronaphthalenones, nodulone B (3) and nodulone C (4) were isolated from Daldinia eschscholtzii associated with terrestrial orchid Paphiopedilum exul (Ridl.) Rolfe, collected from Chiang Mai, Thailand, and display moderate antimicrobial activity. ${ }^{7}$ Furthermore, the isolation of immunosuppressive and antimycobacterial ketodiols such as trans-nodulone $\mathrm{D}(\mathbf{5})^{5}$ and cisnodulone E $(\mathbf{6})^{6}$ from D. eschscholzii and cis- as well as transcorynenones A $\left(\begin{array}{l}7 \\ \text { \& }\end{array}\right)$ from sponge derived Corynespora cassiicola XS-2009017, ${ }^{8}$ suggests that all these metabolites are related and might have similar biogenesis. Moreover, the isolation of both cis- and trans-nodulones from nature suggests the formation of a monoreduced intermediate during their biosynthesis, which has not been isolated as yet. Although, detailed biosynthetic investigations supported by a chemoenzymatic synthesis of related metabolites produced during 1,8dihydroxynaphthalene (DHN) melanin biosynthesis have been performed, ${ }^{9-11}$ the biosynthesis of nodulones remained elusive. ${ }^{7}$ In addition, no synthesis has been reported so far for the preparation of chiral nodulones. It might be due to the presence of the chemically sensitive $\beta$-hydroxyketone group, which can undergo dehydration readily. ${ }^{12-13}$ This motivated us to envisaged a chemoenzymatic, biomimetic synthesis of nodulone A, B and C. The strategy relies on the synthesis of the putative biosynthetic substrates, which on reduction with fungal naphthol reductases may give access to chiral nodulones. Such an approach will provide vital clues about the putative substrates, intermediates, and the enzymes involved in the biosynthesis of nodulones for the first time.

Figure 1. Various natural dihydronaphthalenones isolated from fungul sources. ${ }^{4-8}$

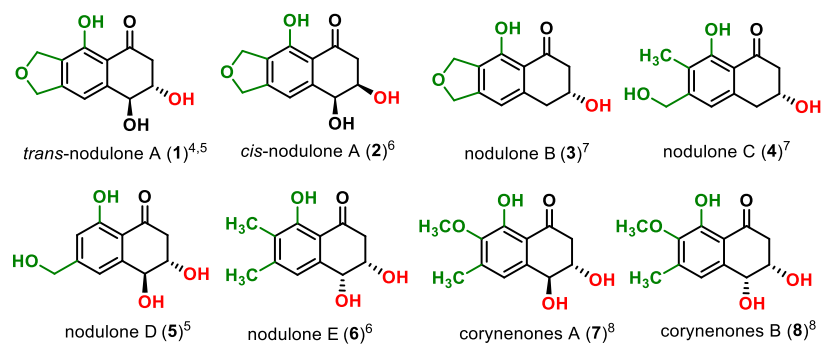

Tetrahydroxynaphthalene and trihydroxynaphthalene reductases ( $\left.\mathrm{T}_{4} \mathrm{HNR} \& \mathrm{~T}_{3} \mathrm{HNR}\right)$ of plant pathogenic fungus Magnaporthe grisea are the two well-studied naphthol reductases involved in DHN-melanin biosynthesis. ${ }^{14-15} \mathrm{~T}_{4} \mathrm{HNR}$ is known to catalyze the NADPH-dependent asymmetric reduction of 1,3,6,8tetrtahydroxynaphthalene $\left(\mathrm{T}_{4} \mathrm{HN}, 9\right)$ to $(R)$-scytalone $(\mathbf{1 0})$ and $\mathrm{T}_{3} \mathrm{HNR}$ catalyzes a similar reduction of $1,6,8$ trihydroxynaphthalene $\left(\mathrm{T}_{3} \mathrm{HN}, \mathbf{1 1}\right)$ to $(R)$-vermelone (12) (Scheme 1). The extended melanin biosynthetic pathway involves the oxidation of $\mathrm{T}_{4} \mathrm{HN}(\mathbf{9})$ and $\mathrm{T}_{3} \mathrm{HN}(\mathbf{1 1})$ to flaviolin (13) and 2hydroxyjuglone (15), respectively. ${ }^{16-18}$ Both, 13 and 15 has been shown to be reduced by $\mathrm{T}_{4} \mathrm{HNR}$ using NADPH to cis-4hydroxyscytalone (14, 4-HS $)^{12}$ and cis-3,4,8-trihydroxytetralone $\left(\mathbf{1 6}, 3,4,8-\right.$ THT$^{13}$ via a mono reduced intermediates (Scheme 1A). ${ }^{18}$ The combination of $\mathrm{T}_{4} \mathrm{HNR}$ and NADPH-regeneration enzyme, glucose dehydrogenase $(\mathrm{GDH})$, or $\mathrm{T}_{3} \mathrm{HNR}$ and GDH results in the formation of trans-4-hydroxyscytalone, indicating the ability of GDH to reduce naphthalenone type compounds. ${ }^{19}$ Over the years, the two naphthol reductases have been shown to catalyze the reduction of several hydroxynaphthoquinones to cis-ketodiols, 
leading to the biomimetic synthesis of several natural products and their analogs. ${ }^{9-11,19}$

Scheme 1. A. T 4 HNR-catalyzed reduction of hydroxynaphthoquinones to cis- and trans-ketodiols. B. Proposed substrates for nodulones.

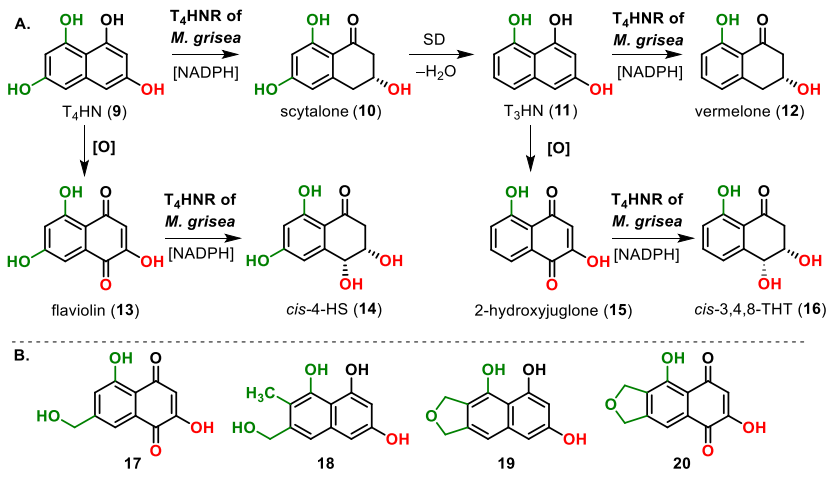

This led us to hypothesize that $\mathrm{T}_{4} \mathrm{HNR}$ and/or $\mathrm{T}_{3} \mathrm{HNR}$ can be used in a chemoenzymatic synthesis of chiral nodulones A, B and C. This would require, at first the chemical synthesis of putative hydroxynaphthalene and hydroxynaphthoquinone substrates. Considering the stereoselective reduction of the known substrates catalyzed by $\mathrm{T}_{4} \mathrm{HNR}$ in particular, ${ }^{9-11}$ we hypothesize that hydroxynaphthoquinone $\mathbf{1 7}$ and hydroxynaphthalene $\mathbf{1 8}$ may act as a substrate for nodulone D (5) and nodulone C (4), respectively. Similarly, $\mathbf{1 9}$ and its oxidized derivative $\mathbf{2 0}$ may act as putative substrates for the (bio)synthesis of nodulone B (3) cis-nodulone A (2), and trans-nodulone A (1) (Scheme 1B). Such an approach may unraveling the steps in volved in the biosynthesis of nodulones in general.

\section{Result and Discussion}

At first, we aimed to synthesize naphthoquinone $\mathbf{1 7}$ which might lead to the preparation of trans-nodulone D (5). For this, ylide 25 was obtained following a two-step synthesis from maleic anhydride and triphenylphosphine (see Supporting Information). ${ }^{20}$ Then a Wittig reaction of 3,5-dimethoxy benzaldehyde (24a) with ylide 25 gave $\mathbf{2 6 a}$ in $60 \%$ yield. The cyclization of $\mathbf{2 6 a}$, followed by aromatization in the presence of potassium acetate in boiling acetic anhydride gave $\mathbf{2 7}$ a in $73 \%$ yield. ${ }^{21}$ This is followed by lithium aluminium hydride reduction of ester to alcohol $\mathbf{2 8}$ in $\mathbf{7 8 \%}$ yield. However, when 28 was treated with strong lewis acid, $\mathrm{BBr}_{3}$ to deprotect methoxy group, the desired product $\mathbf{2 9}$ was not obtained. Therefore, instead of $\mathrm{OMe}$, we used $\mathrm{OAc}$ as protecting group which can be easily deprotected. To make 3,5-diacetoxy benzaldehyde 24b, 3,5-dihydroxy benzoic acid (21) was used as a precursor. Protection of hydroxyl groups in acetic anhydride and a small amount of pyridine gave $\mathbf{2 2 b}$ in $98 \%$ yield. Then $\mathbf{2 2 b}$ was reduced to primary alcohol $\mathbf{2 3 b}$ using borane dimethylsulfate (BMS) in $79 \%$ yield, followed by oxidation to aldehyde $\mathbf{2 4 b}$ using potassium chlorochromate (PCC) in $82 \%$ yield. A Wittig reaction between 24b and ylide 25 gave 26b in $77 \%$ yield. This is followed by cyclization to obtain $\mathbf{2 7} \mathbf{b}$ in $55 \%$ yield. Reduction of $\mathbf{2 7} \mathbf{b}$ with $\mathrm{LAH}$ gave trihydroxynaphthalene $\mathbf{2 9}$, which was oxidized in the presence of $\mathrm{K}_{2} \mathrm{CO}_{3}$ and dimethylformamide (DMF) to obtain desired compound $\mathbf{1 7}$ in $84 \%$ yield. The overall yield after 7 steps was found to be $16 \%$ (Scheme 2).

Next, 17 was used as a substrate for reduction with $\mathrm{T}_{4} \mathrm{HNR}$. For this purpose, the codon-optimized $\mathrm{T}_{4} \mathrm{HNR}$ gene of $M$. grisea cloned into pET19b vector was overexpressed in E.coli BL21 (DE3) to obtain a cell-free extract of $\mathrm{T}_{4} \mathrm{HNR}$-his. ${ }^{10} \mathbf{1 7}$ was then incubated with cell-free lysate of $\mathrm{T}_{4} \mathrm{HNR}$-his and NADPH (regenerated by glucose/glucose dehydrogenase system) in potassium phosphate buffer (50mM, 1mM EDTA, 1mM DTT, pH 7.0) using 2-propanol as co-solvent under anoxic condition for $24 \mathrm{~h}$.

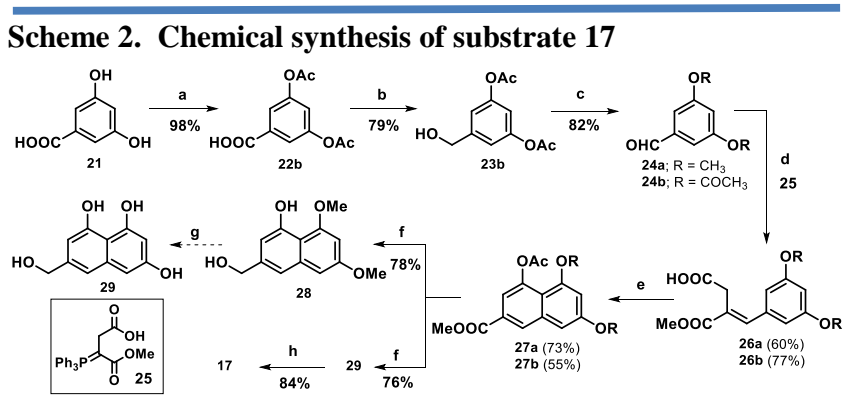

Reagents and conditions: $\left.\left.\mathrm{a}_{1}\right) \mathrm{Ac}_{2} \mathrm{O} / \mathrm{Py}, 70^{\circ} \mathrm{C}, 3 \mathrm{~h} . \mathrm{a}_{2}\right) \mathrm{DMS}, \mathrm{K}_{2} \mathrm{CO}_{3}$, Acetone (dry), reflux, 12h. b) BMS, THF (dry), $0^{\circ} \mathrm{C}$, 30 min. c) PCC, NaOAc, DCM (dry), 1h. d) Benzene (dry), t, 5d. e) $A_{C}$ O, ACOK, reflux, 15 min. f) $L A H$, THF (dry), 2h. g) $\mathrm{BBr}_{3}, \mathrm{DCM}(\mathrm{dry}), 3 \mathrm{~h}$. h) $\mathrm{K}_{2} \mathrm{CO}_{3}$, DMF, 2 h.

The ${ }^{1} \mathrm{H}$ NMR of the crude reaction mixture recorded in acetone- $d_{6}$ shows $62 \%$ conversion. The purification resulted in the isolation of the product in $58 \%$ yield, which was characterized using NMR spectroscopy and mass spectrometry and found to be cis-nodulone $\mathrm{D}(\mathbf{3 0})$ as a major diastereomer along with a small amount of transnodulone $\mathrm{D}(\mathbf{5})$. The $\mathrm{dr}_{\mathrm{cis}} /$ trans was determined to be $95: 5\left({ }^{1} \mathrm{H} \mathrm{NMR}\right)$ (Scheme 3). The absolute configuration of the major diastereomer cis-nodulone $\mathrm{C}$ (30) was assigned by comparing the $\mathrm{CD}$-spectra of the reported cis-3,4,8-trihydroxy-1-tetralone (16) synthesized by the reduction of 2-hydroxyjuglone catalyzed by $\mathrm{T}_{4} \mathrm{HNR}$ (see Supporting Information). ${ }^{11}$ Although, cis-nodulone D has not been isolated as yet from natural sources, we expect it to be a natural product considering the existence of other cis- as well as trans ketodiols.

Since the reduction of $\mathbf{1 7}$ might be a two-step process, we hypothesize the formation of a mono reduced intermediate during the enzymatic transformation. To detect such an intermediate, the transformation was performed only for $4 \mathrm{~h}$. This indeed resulted in the isolation of a diketo intermediate $\mathbf{3 1}$, which is characterized using NMR and HRMS (Scheme 3). Isolation of $\mathbf{3 1}$ indicates that $\mathrm{T}_{4} \mathrm{HNR}$ reduces 17 to $\mathbf{3 1}$ using NADPH, which is then further reduced by either T4HNR or by GDH to give cis-nodulone D as a major diastereomer along with trans-nodulone D. The control experiment, performed using phosphite and cell free lysate of $\mathrm{PTDH}^{22}$ for NADPH regeneration along with $\mathrm{T}_{4} \mathrm{HNR}$ to rule out the role of GDH in the reduction of $\mathbf{3 1}$, surprisingly, gave a mixture of cis- and trans-ketodiol with a dr $\mathrm{c}_{\text {cis/trans }}$ of 80:20 ( ${ }^{1} \mathrm{H}$ NMR). The result suggests that if the reduction of $\mathbf{3 1}$ to cis-nodulone $\mathrm{D}$, most probably catalyzed by $\mathrm{T}_{4} \mathrm{HNR}$ is prevented, we may get transnodulone $\mathrm{D}$ as a major diastereomer.

Scheme 3. T $_{4}$ HNR-catalyzed reduction of substrate 17 to 5

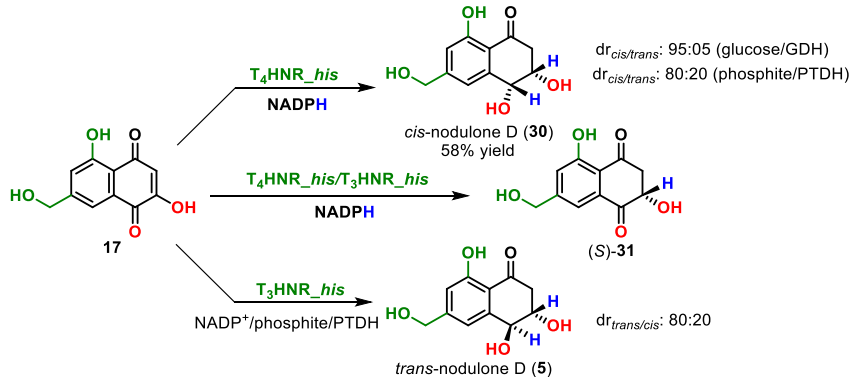

Therefore, we used $\mathrm{T}_{3} \mathrm{HNR}$ of $M$. grisea for the reduction instead of $\mathrm{T}_{4} \mathrm{HNR}$ as it has been reported to give mono reduced product only with similar hydroxynapthoquinone substrates. ${ }^{19}$ For this purpose, the codon-optimized gene of $\mathrm{T}_{3} \mathrm{HNR}$ cloned into the $\mathrm{pET}$ 19 b vector was expressed in $E$. coli BL21 (DE3) to finally obtain the crude lysate. ${ }^{19}$ The incubation of $\mathbf{1 7}$ with crude cell free lysate 
of $\mathrm{T}_{3} \mathrm{HNR}$-his in potassium phosphate buffer ( $\mathrm{pH}$ 7.0) and NADPH (regenerated using phosphite/phosphite dehydrogenase system), gave an inseparable mixture of trans- and cis-nodulone D in the

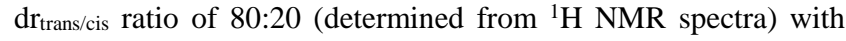
$16 \%$ isolated yield (Scheme 3). A similar transformation performed using NADPH (regenerated using glucose/glucose dehydrogenase system) under the same conditions does not result in the formation of any ketodiol product. The spectral data and sign of optical rotation of trans-nodulone D (5) match that reported in literature ${ }^{5}$, indicating it as same as the natural product (see Supportimg Information). The configuration of trans-nodulone D was further confirmed by CD spectroscopy. Since PTDH shows activity towards the reduction of intermediate 31, we have tested it with 1- and 2-tetralone as substrates, to confirm its ability to reduce a cyclic ketone. Although PTDH shows very low activity towards the reduction of 1-tetralone, we believe that PTDH might have some role in the reduction of $\mathbf{3 1}$ to trans-nodulone D. Further studies will be required to identify the exact role of PTDH in the reduction of $\mathbf{3 1}$.

Scheme 4. A. ThHN-catalyzed reduction of substrate 29 to 32

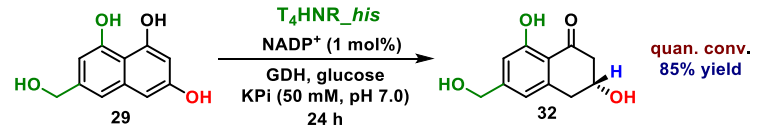

Considering the ability of $\mathrm{T}_{4} \mathrm{HNR}$ to reduce hydroxy naphthalenes, we also subjected compound $\mathbf{2 9}$ for reduction. For this purpose, 29 was incubated with $\mathrm{T}_{4} \mathrm{HNR}$-his and NADPH (regenerated by glucose/GDH system) under the same condition as mentioned above. After $24 \mathrm{~h}$, the reaction mixture shows the formation of dihydronaphthalenone 32 isolated in $85 \%$ isolated yield after purification (Scheme 4). The absolute configuration of $\mathbf{3 2}$ was assigned as $(R)$ by comparison of its CD spectra with that of $(R)$-scytalone reported previously. ${ }^{23}$ Although, no such natural product has been isolated as yet, we propose that $\mathbf{3 2}$ will be isolated in future and probably involve trihydroxynaphthalene $\mathbf{2 9}$ as substrate and $\mathrm{T}_{4} \mathrm{HNR}$ related enzyme for reduction during its biosynthesis.

Next, we aimed to synthesize substrate 18 required for the preparation of nodulone $\mathrm{C}(\mathbf{4})$. For this purpose, 3,5-dihydroxy benzoic acid (21) was converted to MOM protected benzaldehyde 33 in 4steps through protection, reduction and oxidation (see Supporting Information) (Scheme 5). ${ }^{24}$ Then, aldehyde 33 was used in Stobbe condensation with methyl succinic ester 34 in the presence of $\mathrm{NaH}$ which lead to the isolation of acid $\mathbf{3 5}$ in $20 \%$ yield. 34 was synthesized by esterification of methyl succinic acid. Low yield in this reaction, was due to the Cannizaro reaction ${ }^{25}$ of aldehyde 33 which took place in presence of sodium hydride and resulted in the formation of side products, acid and alcohol (see supporting information).

\section{Scheme 5. Chemical synthesis of substrate 18}

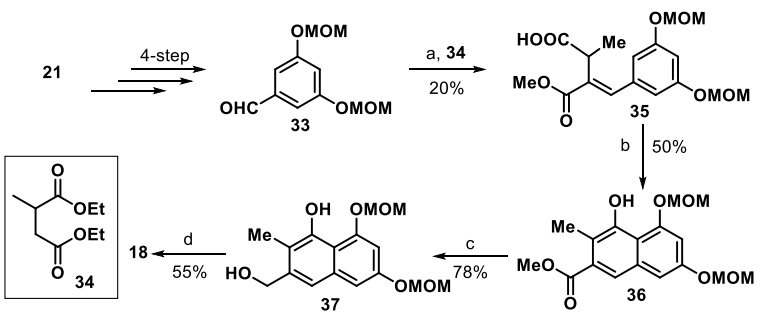

Reagents and conditions: (a) NaH, Toluene (dry), cat. EtOH, rt, 1h. (b) $\mathrm{Ac}_{2} \mathrm{O}, \mathrm{AcOK}$, reflux, 15 min. (c) $L A H, T H F(d r y), 0^{\circ} \mathrm{C}, 1 \mathrm{~h}$. (d) $\mathrm{MeOH}, \mathrm{HCL}(\mathrm{c}), 60^{\circ} \mathrm{C}, 30 \mathrm{~min}$.
Despite optimization efforts, the yield of Stobbe condensation could not be improved further. Nevertheless, the cyclisation and aromatisation of $\mathbf{3 5}$ using potassium acetate in boiling acetic acid gave 36 in 50\% isolated yield. Finally, the desired substrate $\mathbf{1 8}$ was obtained in a two-step process from $\mathbf{3 6}$, involving reduction using $\mathrm{LAH}$, followed by the deprotection of compound $\mathbf{3 7}$ by heating in $\mathrm{MeOH}$ in presence of acid (Scheme 5). Then, hydroxynaphthalene 18 was incubated with $T_{4} H N R$ using NADPH (regenerated using glucose/glucose dehydrogenase as a co-factor in potassium phosphate buffer (50mM, 1mM EDTA, 1mM DTT, pH 7.0) for $24 \mathrm{~h}$ under anoxic conditions. After purification, we got the desired nodulone C (4) in 90\% isolated yield (Scheme 6). 4 was characterized using NMR spectroscopy and mass spectrometry. The comparison of NMR data and optical rotation confirmed it to be identical to the naturally occurring nodulone C (4). Absolute configuration of the chiral centre was assigned by comparing the CD spectra of $(R)$-scytalone synthesized previously using $\mathrm{T}_{4} \mathrm{HNR}^{23}$

\section{Scheme 6. T4HN-catalyzed reduction of substrate 18 to 4}

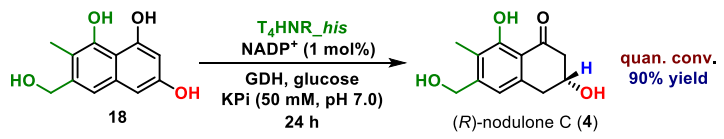

Next, we also attempted the synthesis of the substrates $\mathbf{1 9}$ and $\mathbf{2 0}$ required for the preparation of furan containing nodulones $\mathrm{A}$ and B. For this, we designed a retrosynthetic strategy and according to which we first made ester $\mathbf{4 0}$ by [4+2] cycloaddition from furan (38) and dimethyl acetylenedicarboxylate (DMAD, 39) in $45 \%$ yield (Scheme 7). Then, a single carbon-carbon double bond is reduced by $\mathrm{H}_{2}, \mathrm{Pd} / \mathrm{C}$ in $85 \%$ yield, followed by retro-Diels-Alder reaction to obtain $\mathbf{4 2}$ in $96 \%$ yield. The diester $\mathbf{4 2}$ was then reduced using $\mathrm{LiAlH}_{4}$ in $80 \%$ yield to obtain $\mathbf{4 3}$. Despite several attempts, we were unable to get furofuran $\mathbf{4 4}$ from $\mathbf{4 3}$, which we plan to use for another Diels-Alder reaction with 45 to obtain 46, which on deprotection might have given us the desired substrate 19 and 20 (Scheme 7).

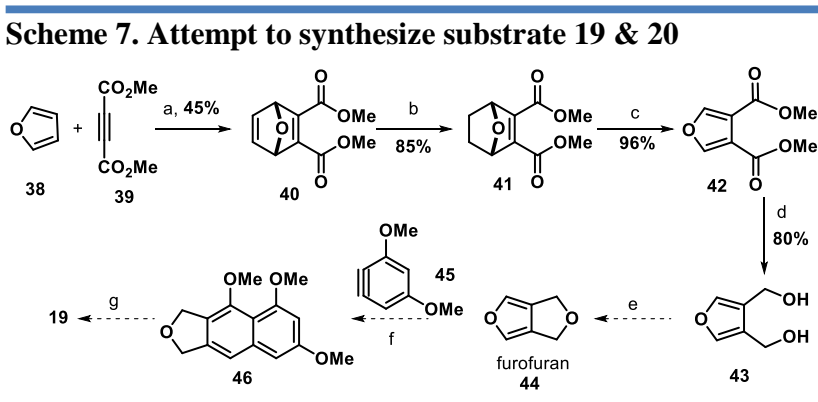

Reagents and conditions: (a) $100^{\circ} \mathrm{C}, 18 \mathrm{~h}$. (b) $10 \% \mathrm{Pd} / \mathrm{C}, \mathrm{H}_{2}$ 6h. (c) $175^{\circ} \mathrm{C}, 1 \mathrm{~h}$. (d) $\mathrm{LiAlH}_{4}$, Ether (dry), $0^{\circ} \mathrm{C}-\mathrm{rt}, 1 \mathrm{~h}$. (e) $\mathrm{MnO}_{2}$, Triethylsilane, TFA, DCM $\left(\left(-5^{\circ} \mathrm{C}\right.\right.$ to rt $)$

Nevertheless, based on the earlier studies on related natural products and our chemoenzymatic synthesis of nodulone $\mathrm{C}$ and $\mathrm{D}$ presented here, we proposed the following biosynthetic pathway for the formation of nodulones. In previous work, ${ }^{7}$ trans-nodulone $\mathrm{D}$ was proposed to be formed by the decarboxylation followed by reduction of hydroxynaphthoquinone $\mathbf{3 9}$, formed by the cyclization of a linear polyketide 47 (Path A, Scheme 7). Alternatively, we propose the formation of compound $\mathbf{4 9}$ by the regioselective cyclization, which on decarboxylation will give substrate $\mathbf{2 9}$, which will be reduced to give compound $\mathbf{3 2}$, which is yet to be isolated from natural sources (Path B, Scheme 7). We propose, that 29 on oxidation will form hydroxynaphthoquinone $\mathbf{1 7}$, similar to the oxidation of $\mathrm{T}_{4} \mathrm{HN}$ to flaviolin. This will then undergo reduction 
catalyzed by $\mathrm{T}_{4} \mathrm{HNR}$ related enzyme to form either cis-nodulone D (30), which is not yet isolated or trans-nodulone (5) which is a known natural product. Furthermore, 49 may also form 18 by reduction of carboxylic acid, which on reduction by naphthol reductase related enzyme(s) will form nodulone C (4).

\section{Scheme 8. Proposed biosynthesis of nodulones A-D}

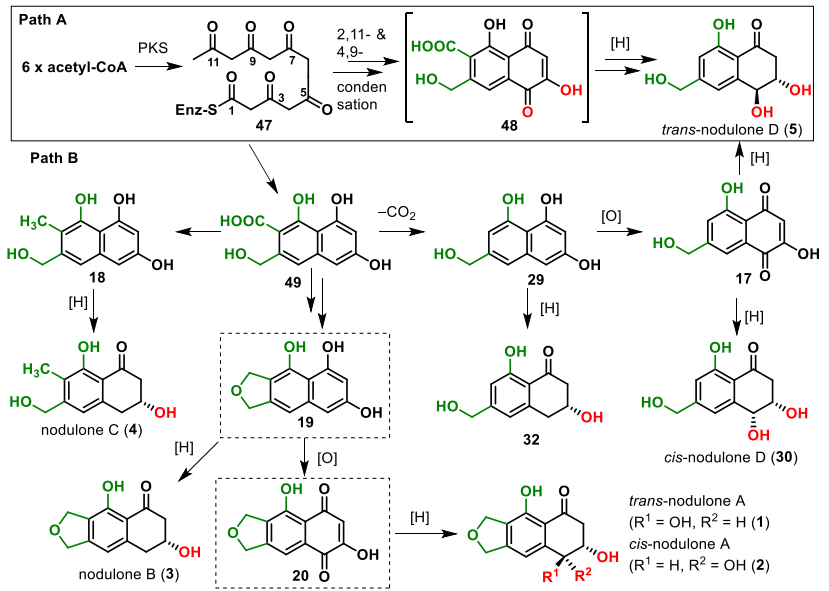

Although the putative substrates 19 and $\mathbf{2 0}$ could not be synthesized and tested with the reductase as yet, we propsoe that reduction and cyclization of $\mathbf{4 9}$ will form furano naphthalene substrate, which on reduction catalyzed by $\mathrm{T}_{4} \mathrm{HNR}$ related enzyme will form nodulone $\mathrm{B}$ (3) and on oxidation will give hydroxynaphthoquinone $\mathbf{2 0}$ which on reduction by the enzyme will lead to the formation of cis- as well as trans-nodulone A $(\mathbf{1} \& \mathbf{2})$. This needs to be validated in future by the reduction of substrate $\mathbf{2 0}$ using a naphthol reductase.

In summary, we report here the first asymmetric synthesis of $(R)$ nodulone C (4) and trans-nodulone D (5) using a chemoenzymatic approach. Although we could not synthesize the furan naphthalene substrate proposed for the preparation of cis- and trans-nodulone A, we speculate that their biosynthesis would involve a similar strategy for its formation. In addition, we have obtained cisnodulone D (30) and dihydronapthalenone 32 after the enzymatic reduction, which we propose to be natural products and may be isolated in future. Our results further corroborate the broad substrate scope shown by $\mathrm{T}_{4} \mathrm{HNR}$ and $\mathrm{T}_{3} \mathrm{HNR}$ and their involvement in the biosynthesis of several fungal metabolites. Finally, based on the chemoenzymatic approach a probable biosynthetic pathway $\mathrm{B}$ has been proposed for the formation of various nodulones, which further shows the ability of nature to create molecular diversity through branching achieved through oxidation and reduction reactions during biosynthesis.

\section{ASSOCIATED CONTENT}

\section{Supporting Information}

The Supporting Information is available free of charge on the ACS Publications website.

Protein expression and purification, experimental details, characterization data, copies of NMR spectra, and CD-spectra (PDF).

\section{AUTHOR INFORMATION}

\section{Corresponding Author}

*E-mail: smhusain@cbmr.res.in, smhusain.cbmr@gmail.com Notes

The authors declare no competing financial interest.

\section{ACKNOWLEDGEMENT}

We are grateful to the Council of Scientific and Industrial Research, New Delhi (Project no. 02(0258)/16/EMR-II) and SERB (CRG/2018/002682) for funding and CSIR, New Delhi for funding fellowships to T. M. and N. S. We are thankful to Prof. Werner Hummel for providing the plasmid of glucose dehydrogenase and the Director, Centre of Biomedical Research for research facilities.

\section{REFERENCES}

(1) Cruz, J. S.; da Silva, C. A.; Hamerski, L J. Fungi. 2020, 6, 128155 .

(2) Nisa, H.; Kamili, A. N.; Nawchoo, I. A.; Shafi, S.; Shameem, N.; Bandh, S. A. A review. Microb Pathog. 2015, 82, 50-59.

(3) Kaul, S.; Gupta, S.; Ahmed, M.; Dhar, M. K. Phytochem Rev. 2012, 11, 487-505.

(4) Wu, Z.-C.; Li, D.-L.; Chen, Y.-C.; Zhang, W.-M. Helv. Chim Acta. 2010, 93, 920-923.

(5) Zhang, Y. L.; Zhang, J.; Jiang, N.; Lu, Y. H.; Wang, L.; Xu, S. H.; Wang, W.; Zhang, G. F.; Xu, Q.; Ge, H. M.; et al. J. Am. Chem. Soc. 2011, 133, 5931-5940.

(6) Prabpai, S.; Wiyakrutta, S.; Sriubolmas, N.; Kongsaeree, P. Phytochem. Lett. 2015, 13, 375-378.

(7) Barnes, E. C.; Jumpathong, J.; Lumyong, S.; Voigt, K.; Hertweck, C. Chem. Eur. J. 2016, 22, 4551-4555.

(8) Zhao, D-L.; Shao, C-L.; Wang, C-Y.;Wang, M.; Yang, L-J.; Wang, C-Y. Molecules. 2016, 21, 160-165.

(9) Husain, S. M.; Schätzle, M. A.; Röhr, C.; Lüdeke, S.; Müller, M. Org. Lett. 2012, 14, 3600-3603.

(10) Husain, S. M.; Schätzle, M. A.; Lüdeke, S.; Müller, M. Angew. Chem. Int. Ed. 2014, 53, 9806-9811.

(11) Saha, N.; Müller, M.; Husain, S. M. Org. Lett. 2019, 21, 2204-2208.

(12) Couche', E.; Fkyerat, A.; Tabacchi, R. Helv. Chim. Acta. 2009, 92, 903-917.

(13) Mukherjee, P.; Roy, S. J. S.; Sarkar, T. K. Org. Lett. 2010, 12, 2472-2475.

(14) Thompson, J. E.; Fahnestock, S.; Farrall, L.; Liao, D.-I.; Valent, B.; Jordan, D. B. J. Biol. Chem. 2000, 275, 1740-1745.

(15) Vidal-Cros, A.; Viviani, F.; Labesse, G.; Boccara, M.; Gaudry, M. Eur. J. Biochem. 1994, 219, 985-992.

(16) Bell, A. A.; Wheeler, M. H. Annu. Rev. Phytopathol. 1986, 24, 411-451.

(17) Abou-Mansour, E.; Couché, E.; Tabacchi, R. Phytopathol. Mediterr. 2004, 43, 75-82.

(18) Husain, S.; Müller, M. Synlett. 2017, 28, 2360-2372.

(19) Conradt, D.; Schätzle, M. A.; Husain, S. M.; Müller, M. ChemCatChem. 2015, 7, 3116-3120.

(20) Doulut, S.; Dubuc, I.; Rodriguez, M.; Vecchini, F.; Fulcrand, H.; Barelli, H.; Checler, F.; Bourdel, E.; Aumelas, A. Lallement, J. C.; Kitabgi, P.; Costentin, J.; Martinez, J. J. Med. Chem. 1993, 36, 1369-1379.

(21) Piettre, A.; Chevenier, E.; Massardier, C.; Gimbert, Y.; Greene, A. E. Org. Lett. 2002, 4, 3139-3142.

(22) Watanabe, H.; Hirakawa, H.; Nagamune, T. ChemCatChem. 2013, 5, 3835-3840.

(23) Schätzle, M. A.; Flemming, S.; Husain, S. M.; Richter, M.; Günther, S.; Müller, M. Angew. Chem., Int. Ed. 2012, 51, 2643 2646.

(24) Sengupta, S.; Bae, M.; Oh, D.-C.; Dash, U.; Kim, H. J.; Song, W. Y.; Shin, I.; Sim, T. J. Org. Chem. 2017, 82, 12947-12966.

(25) Jiang, X.-D.; Matsukawa, S.; Kakuda, K.; Fukuzaki, Y.; Zhao, W.-L.; Li, L.-S.; Shen, H. -B.; Kojima, S.; Yamamoto, Y. Dalton Trans. 2010, 39(41), 9823-9829. 